\title{
Dolor abdominal agudo y obstrucción intestinal como presentación clínica de intoxicación por plomo: reporte de caso
}

\author{
Guadalupe E. Gavilánez-Chávez ${ }^{1 *}$, Jordy Millán-Lizárraga1', Luis A. Pulido y Harumi Scott-López² \\ ${ }^{1}$ Servicio de Urgencias, Hospital General Regional 46, Instituto Mexicano del Seguro Social (IMSS); ${ }^{2}$ Laboratorio Regional de Salud en el Trabajo, \\ Centro Medico Nacional de Occidente, IMSS. Guadalajara, Jalisco, México
}

\begin{abstract}
Resumen
El dolor abdominal agudo y la obstrucción intestinal son padecimientos frecuentes en el servicio de urgencias, en ambos casos la gama de posibilidades diagnosticas es amplia, desde condiciones benignas hasta las potencialmente mortales, uno de los diagnósticos diferenciales es la intoxicación por plomo. Presentamos el caso de un varón de 28 años con dolor abdominal agudo y obstrucción intestinal que se dedica a la extracción-fundición de plomo a partir de baterías recicladas, el nivel de plomo encontrado fue de $86.32 \mu \mathrm{g} / \mathrm{dl}$ y $40.59 \mu \mathrm{g} / \mathrm{dl}$ posterior al tratamiento a base de $d$-penicilamina. Los pacientes con toxicidad aguda por plomo con manifestaciones gastrointestinales se presentan con síntomas inespecíficos. Ante un paciente con dolor abdominal y obstrucción intestinal se debe pensar en esta causa como diagnóstico diferencial sobre todo en jóvenes con antecedente de exposición.
\end{abstract}

Palabras clave: Dolor abdominal. Obstrucción intestinal. Intoxicación por plomo. Saturnismo.

Acute abdominal pain and bowel obstruction as a clinical presentation of lead poisoning: a case report

\begin{abstract}
Acute abdominal pain and bowel obstruction are frequent conditions in the emergency department, in both cases the range of diagnostic possibilities is wide, from benign to life-threatening conditions, one of the differential diagnosis is lead poisoning. We present the case of a 28-year-old man with acute abdominal pain and bowel obstruction. He is dedicated to the extraction-smelting of lead from recycled batteries, the lead level found was $86.32 \mu \mathrm{g} / \mathrm{dl}$ and $40.59 \mu \mathrm{g} / \mathrm{dl}$ after treatment based on $d$-penicillamine. Patients with acute lead toxicity with gastrointestinal manifestations will present with non-specific symptoms. To a patient with abdominal pain and bowel obstruction this cause should be thought of as a differential diagnosis, especially in young people with a history of exposure.
\end{abstract}

Keywords: Abdominal pain. Bowel obstruction. Lead toxicity. Plumbism.

\section{Correspondencia:}

*Guadalupe E. Gavilánez-Chávez E-mail: g_gavilanez@ hotmail.com
Fecha de recepción: 10-08-2021

Fecha de aceptación: 02-11-2021 DOI: $10.24875 / R E I E .21000087$
Disponible en internet: 14-02-2022

Rev Educ Investig Emer. 2022;4(1):50-54 www.medicinadeemergencias.com

2604-6520 @ 2022 Sociedad Mexicana de Medicina de Emergencias, AC. Publicado por Permanyer México SA de CV. Este es un artículo open access bajo la licencia CC BY-NC-ND (http://creativecommons.org/licenses/by-nc-nd/4.0/). 


\section{Introducción}

El dolor abdominal agudo es un padecimiento frecuente en el servicio de urgencias, que representa el $16 \%$ de los motivos de atención ${ }^{1}$. Por su parte, la obstrucción intestinal es el motivo del $20 \%$ de las admisiones quirúrgicas por un problema abdominal agudo ${ }^{2}$. En ambos casos, la gama de posibilidades diagnósticas al ingreso es amplia y se extiende desde condiciones benignas hasta las potencialmente mortales. Uno de los diagnósticos diferenciales es la intoxicación por plomo ${ }^{3}$. Esta es una causa poco común que puede presentarse con signos y síntomas poco específicos, como dolor abdominal intenso y recurrente, constipación, irritabilidad, náusea y vómito ${ }^{4}$; otros síntomas son polineuropatía, debilidad y mialgias ${ }^{5}$. Además de los síntomas gastrointestinales, la toxicidad aguda puede ser manifestada por insuficiencia renal y encefalopatía ${ }^{6}$.

La exposición laboral al plomo es una de las principales fuentes de intoxicación ${ }^{7}$. Se identifican con mayor riesgo los mineros, los fundidores de plomo, los fabricantes y recicladores de baterías, los fontaneros, los mecánicos de automóviles, los fabricantes de vidrio, los trabajadores de la construcción, los instructores de campo de tiro, los fabricantes de plástico y los soldadores ${ }^{8}$.

Este caso clínico sigue las directrices CARE ${ }^{9}$.

\section{Presentación del caso}

Varón de 28 años que se dedica a la extracción-fundición de plomo a partir de baterías recicladas desde hace 6 meses, con tabaquismo positivo activo de 20 cigarrillos diarios durante 10 años, que acude al servicio de urgencias por un cuadro de 5 días de evolución caracterizado por dolor abdominal agudo, ausencia de evacuaciones, sin canalizar gases, fatiga extrema y dolor lumbar y de extremidades inferiores. A su ingreso a nuestro hospital muestra los siguientes signos vitales: presión arterial 110/70 mmHg, frecuencia cardiaca $80 /$ min, frecuencia respiratoria $16 /$ min y saturación de oxígeno $94 \%$. Presenta facies franca de dolor abdominal, neurológico íntegro, sin afectación de la fuerza y la sensibilidad, palidez marcada de piel y tegumentos, abdomen blando, depresible, doloroso en todo el trayecto colónico, peristalsis incrementada, signo de Blumberg positivo, y al tacto rectal ámpula vacía, sin masas ni tumoraciones.

En la radiografía simple de abdomen se observan asas intestinales distendidas, de predominio en el

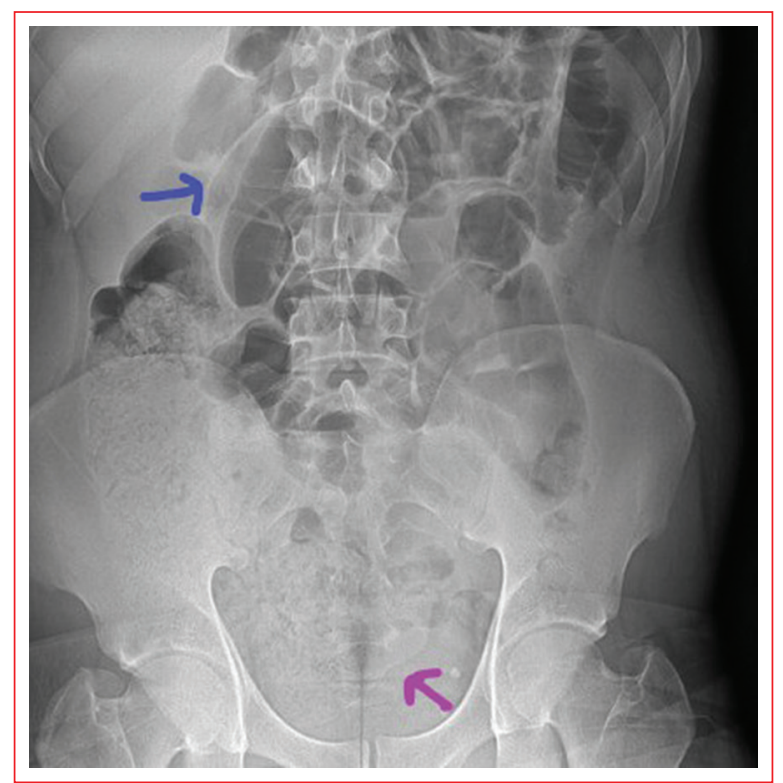

Figura 1. Radiografía simple de abdomen. La flecha azul señala asas de intestino delgado dilatadas con edema entre ellas. La flecha morada señala la ámpula rectal sin distribución de gas ni residuo fecal, sugestivo de oclusión intestinal.

intestino delgado, sugestivas de oclusión intestinal alta sin delimitar el sitio ni la causa aparente. Se recomienda estudio tomográfico para determinar lo antes mencionado (Fig. 1).

La tomografía de abdomen evidencia un aumento del patrón neumofecal en el colon ascendente y el ciego en relación con coprostasis, aumento de calibre de las asas en el colon transverso descendente y el intestino delgado con abundante aire en su interior, y ausencia de gas en la ámpula rectal, concluyendo hallazgos sugestivos de obstrucción intestinal sin evidencia de sitio de transición (Figs. 2 y 3).

La biometría hemática mostró hemoglobina 12.8, hematocrito 37 , volumen corpuscular medio 89 , hemoglobina corpuscular media 30, plaquetas 147,000, leucocitos 10,000, glucosa 101, urea 32 , creatinina 0.7 , tiempo de protrombina 14/12, International Normalized Ratio 1.32, tiempo parcial de tromboplastina $32 / 30$, calcio 9.1 , cloro 101 , potasio 3.9 , sodio 137 , magnesio 1.54 , amilasa 32 y lipasa 15 , con ligera elevación de las pruebas funcionales hepáticas (alanina aminotransferasa 58 , aspartato transaminasa 45) y deshidrogenasa láctica 244. La cuantificación del plomo se realizó en el Laboratorio Regional de Salud en el Trabajo y Ambiental del Centro Médico Nacional de Occidente, del Instituto Mexicano del 
Tabla 1. Resultado de la determinación analítica de plomo en sangre

\begin{tabular}{|l|l|l|l|l|}
\hline Numero de muestra & Matriz biológica & Analito & Método de ensayo & Resultados \\
\hline $\begin{array}{l}\text { Primera } \\
\text { (antes del tratamiento) }\end{array}$ & Sangre & Plomo & $\begin{array}{l}\text { Espectroscopía de absorción atómica, horno de } \\
\text { grafito }\end{array}$ & $86.32 \mu \mathrm{g} / \mathrm{dl}$ \\
\hline $\begin{array}{l}\text { Segunda } \\
\text { (después del tratamiento) }\end{array}$ & Sangre & Plomo & $\begin{array}{l}\text { Espectroscopía de absorción atómica, horno de } \\
\text { grafito }\end{array}$ & $40.59 \mu \mathrm{g} / \mathrm{dl}$ \\
\hline $\begin{array}{l}\text { Valores de referencia en } \\
\text { personal expuesto laboralmente }\end{array}$ & & & $<30 \mu \mathrm{g} / \mathrm{dl}$ \\
\hline \begin{tabular}{l} 
Referencias: NOM 0-47-SSA1-2011 y NOM-199-SSA1-2000. \\
\hline
\end{tabular} & & & \\
\hline
\end{tabular}

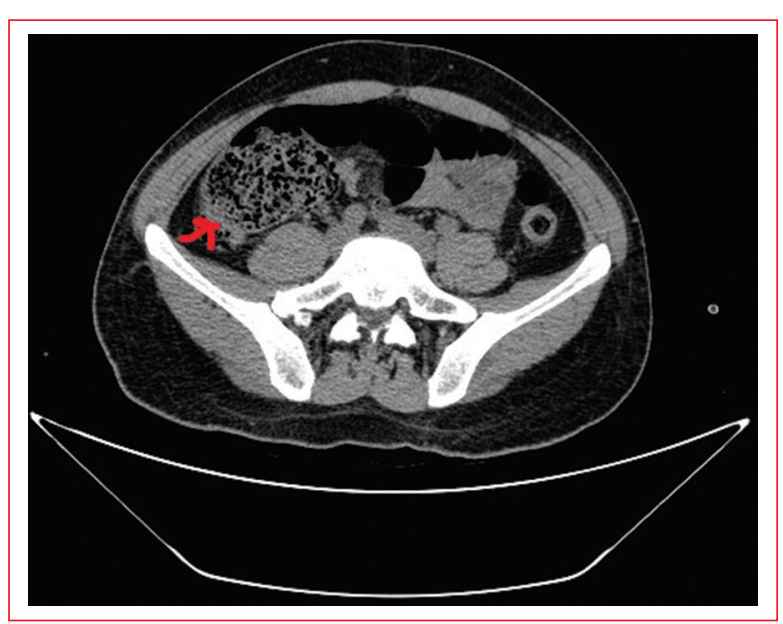

Figura 2. Tomografía simple de abdomen, corte axial. La flecha roja señala aumento del patrón neumofecal en el colon ascendente y el ciego, en relación con coprostasis.

Seguro Social, mediante espectrofotometría de absorción atómica con horno de grafito, que reportó una plumbemia de $86.32 \mu \mathrm{g} / \mathrm{dl}$ (Tabla 1). Ante los antecedentes de exposición laboral y con los hallazgos clínico-radiológicos y analíticos se estableció el siguiente tratamiento: sonda nasogástrica a derivación, ketorolaco $30 \mathrm{mg}$ por vía intravenosa cada 12 horas, buprenorfina $150 \mu \mathrm{g}$ por vía subcutánea por razón necesaria y ondansetrón $8 \mathrm{mg}$ por vía intravenosa cada 12 horas. También se inició D-penicilamina a dosis de $300 \mathrm{mg}$ por vía oral cada 24 horas por 4 semanas. El paciente se mantuvo en vigilancia intrahospitalaria por 7 días, logrando evacuación a los 6 días de su ingreso y remitiendo la sintomatología. Diez semanas después, en las que el paciente se mantuvo libre de exposición y asintomático, se le realizó una nueva determinación de plomo que reportó $40.59 \mu \mathrm{g} / \mathrm{dl}$ (Tabla 1).

\section{Discusión}

Refiriéndonos específicamente a los fundidores de plomo, el tiempo mínimo de exposición laboral para el desarrollo de síntomas de plumbemia se ha establecido incluso en 30 días, y el grupo de edad más afectado en trabajadores expuestos a fundición es el de 25-29 años ${ }^{10}$.

La determinación de plomo en sangre venosa es la prueba más sensible de exposición al plomo ${ }^{11}$, en especial cuando se realiza por espectrofotometría de absorción atómica con horno de grafito ${ }^{6}$.

En la actualidad, en nuestro país se recomienda que los niveles de plomo en sangre de personal laboralmente expuesto se mantengan por debajo de $30 \mu \mathrm{g} / \mathrm{dl}$; en personal no expuesto laboralmente, en menos de $10 \mu \mathrm{g} / \mathrm{dl}$; y en niños y mujeres embarazadas o en periodo de lactancia en menos de $5 \mu \mathrm{g} / \mathrm{dl}^{12}$. No obstante, de modo mas estricto la Organización Mundial de la Salud sugiere que no existen concentraciones de plomo que puedan considerarse seguras ${ }^{6}$, debido a que se ha demostrado que niveles considerados como seguros pueden provocar problemas cognitivos, trastornos neuroconductuales, diferentes daños neurológicos, hipertensión arterial e insuficiencia renal ${ }^{13}$.

El tratamiento con agentes quelantes se recomienda cuando los niveles de plomo están por encima de $45 \mu \mathrm{g} / \mathrm{dl}^{14}$. Se recomiendan edetato disódico cálcico (EDTA), dimercaprol, D-penicilamina y succimer (ácido 2,3-dimercaptosuccínico) ${ }^{15}$.

Para la D-penicilamina, la dosis inicial es de $10 \mathrm{mg} / \mathrm{kg} / \mathrm{día}$ divididos cada 12 horas, por vía oral, durante 2 semanas, incrementando a $25 \mathrm{mg} / \mathrm{kg} /$ día (máximo $1.5 \mathrm{~g} / \mathrm{día}$ ), administrándola 1 hora antes o 3 horas después de los alimentos, durante 4 a 8 semanas, con un segundo control de los niveles de plomo a las 4 semanas ${ }^{16,17}$. 


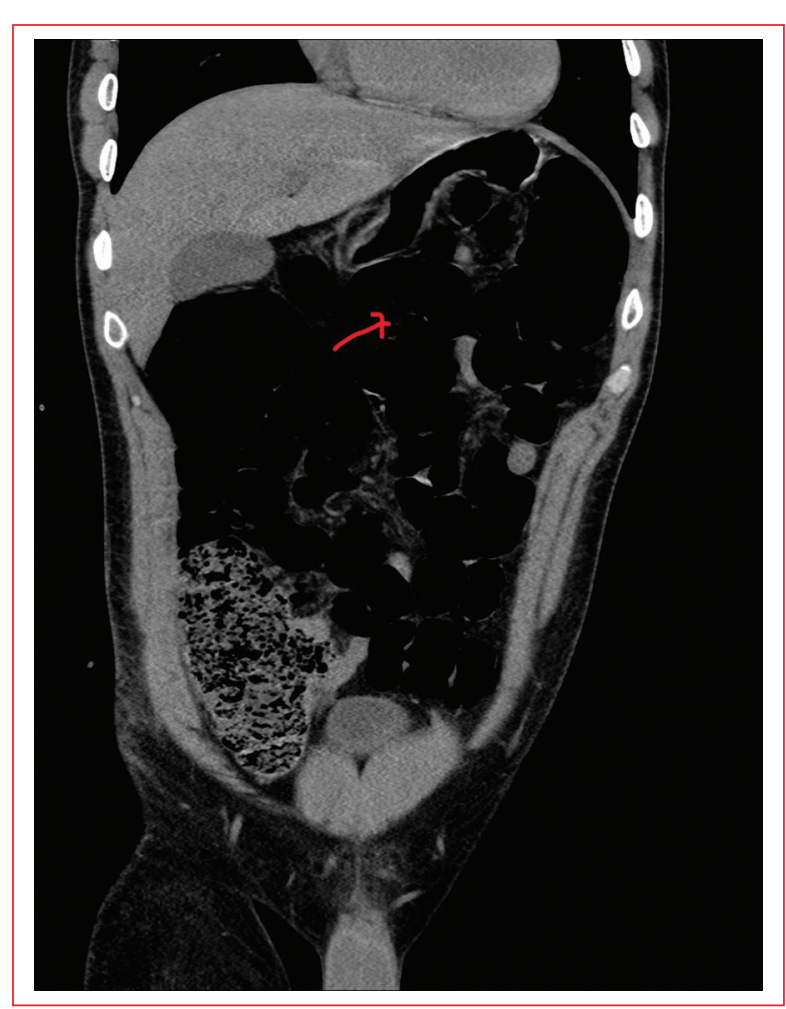

Figura 3. Tomografía simple de abdomen, corte coronal. La flecha roja señala aumento de calibre de las asas en el colon transverso-descendente y el intestino delgado, con abundante aire en su interior, sugestivo de obstrucción intestinal.

Ademas del tratamiento sintomático y quelante, una importante medida es retirar al trabajador de la fuente de exposicion hasta que los niveles de plomo en sangre sean menores de $40 \mu \mathrm{g} / \mathrm{dl}^{18}$.

\section{Conclusiones}

La mayoría de los pacientes con toxicidad aguda por plomo se presentan con síntomas gastrointestinales inespecíficos. El saturnismo debe ser considerado como causa de dolor y obstrucción intestinal sobre todo en pacientes jóvenes con antecedente de exposición.

\section{Agradecimientos}

A María Erika Ramos Hernández, del laboratorio de análisis clínicos del Hospital General Regional No. 46 del Instituto Mexicano del Seguro Social. A Itzell Reyes García, médico radiólogo del Hospital General Regional No. 46 del Instituto Mexicano del Seguro Social. Al equipo de médicos urgenciólogos y cirujanos que participaron en el correcto abordaje del paciente en el servicio de urgencias del Hospital General Regional No. 46 del Instituto Mexicano del Seguro Social.

\section{Financiamiento}

Los gastos generados en la elaboración del manuscrito estuvieron a cargo de los investigadores.

\section{Conflicto de intereses}

Los autores declaran no tener conflictos de intereses.

\section{Responsabilidades éticas}

Protección de personas y animales. Los autores declaran que para esta investigación no se han realizado experimentos en seres humanos ni en animales.

Confidencialidad de los datos. Los autores declaran que han seguido los protocolos de su centro de trabajo sobre la publicación de datos de pacientes.

Derecho a la privacidad y consentimiento informado. Los autores han obtenido el consentimiento informado de los pacientes y/o sujetos referidos en el artículo. Este documento obra en poder del autor de correspondencia.

\section{Bibliografía}

1. Sebbane M, Dumont R, Jreige R, Eledjam J. CT of the Acute Abdomen. Epidemiology of Acute Abdominal Pain in Adults in the Emergency Department Setting. Berlin, Heidelberg: Springer; 2011. p. 3-13.

2. Domínguez GE. Factores predictivos de mortalidad en la oclusión intestinal por bridas. Repertorio de Medicina y Cirugía. 2016;25:163-7.

3. Shabani M, Hadeiy SK, Parhizgar P, Zamani N, Mehrad H, Hassanian-Moghaddam $\mathrm{H}$, et al. Lead poisoning; a neglected potential diagnosis in abdominal pain. BMC Gastroenterol. 2020;20:134.

4. Chang SH, Yoon SB, Lee JW, Lee DJ. What caused hemolytic anemia and colicky abdominal pain? ¡Lead! Korean J Intern Med. 2013;28:504-6.

5. Dequanter D, Lefebvre JC, Takieddine M, Belva P, Vaneukem P. An acute pseudo-cholecystitis. Rev Med Brux. 2001;22:439-41.

6. Azcona-Cruz MI, Ramírez y Ayala R, Vicente-Flores G. Efectos tóxicos del plomo, Articulo de revisión. Rev Esp Med Quir. 2015;20:72-7.

7. Wani AL, Ara A, Usmani JA. Lead toxicity: a review. Interdiscip Toxicol. 2015;8:55-64.

8. Sanborn MD, Abelsohn A, Campbell M, Weir E. Identifying and managing adverse environmental health effects: 3 . Lead exposure. CMAJ. 2002;166:1287-92.

9. Riley DS, Barber MS, Kienle GS, Aronson JK, von Schoen-Angerer T, Tugwell $P$, et al. CARE explanation and elaborations: reporting guidelines for case reports. J Clin Epidemiol. 2017:89:218-35.

10. Carlin CE, Ferrandiz JJ. Algunas consideraciones sobre saturnismo entre los trabajadores de una fundición. Rev Peru Med Exp Salud Publica. 1956;10:43-52.

11. Shiri R, Ansari M, Ranta M, Falah-Hassani K. Lead poisoning and recurrent abdominal pain. Ind Health. 2007;45:494-6.

12. Norma Oficial Mexicana NOM 047-SSAA1-2011. Salud ambiental, índices Salud ambiental, índices biológicos de exposición para personal ocupacionalmente expuesto a sustancias quimicas. Disponible en http:// www.dof.gob.mx/. 
Rev Educ Investig Emer. 2022;4(1)

13. Begovic V, Nozic D, Kupresanin S, Tarabar D. Extreme gastric dilation caused by chronic lead poisoning: a case report. World J Gastroenterol. 2008; 14:2599-601.

14. Centers for Disease Control and Prevention (CDC). Advisory Committee on Childhood Lead Poisoning Prevention. Low level lead exposure harms children: a renewed call for primary prevention. Atlanta, GA: US Department of Health and Human Services, CDC, Advisory Committee on Childhood Lead Poisoning Prevention; 2012. (Consultado en agosto de 2012.) Disponible en: http://www.cdc.gov/nceh/lead/acclpp/final_document_010412.pdf

15. Poma PA. Lead effects on humans. An Fac Med. 2008;69:120-6.
16. Fontana D, Lascano VM, Solá N, Martínez S, Virgolinni M, Mazzieri MR Intoxicación por plomo y su tratamiento farmacológico. Revista de Salud Pública. 2013;17:49-59.

17. Paeezi M, Zamani N, Hassanian-Moghaddam H, Shadnia S, Zamani N, Chaleshi V, et al. Treatment of adult lead poisoning with D-penicillamine. Drug Metab Pers Ther. 2019;34:/j/dmdi.2019.34.issue-2/dmpt-20190003/dmpt-2019-0003.xml.

18. National Institute for Ocupational Safety and Health (NIOSH). Reducing exposure to lead and noise at outdoor firing ranges. Publication $N$ ․o $2013-$ 104. November 2012. 